\title{
Breast cancer risk and imprinting methylation in blood
}

\author{
Kristina Harrison', Gwen Hoad ${ }^{1}$, Paula Scott', Louise Simpson², Graham W. Horgan², Elizabeth Smyth", \\ Steven D. Heys ${ }^{2}$ and Paul Haggarty ${ }^{1 *}$
}

\begin{abstract}
Background: Altered DNA methylation of imprinted genes has been implicated in a range of cancers. Imprinting is established early in development, and some are maintained throughout the life course in multiple tissues, providing a plausible mechanism linking known early life factors to cancer risk. This study investigated methylation status of seven imprinted differentially methylated regions_PLAGL1/ZAC1, H19-ICR1, IGF2-DMR2, KvDMR-ICR2, RB1, SNRPN-DMR1 and PEG3 - in blood samples from 189 women with the most common type of invasive breast cancer (invasive ductal carcinoma-IDC), 41 women with in situ breast cancer (ductal carcinoma in situ—DCIS) and 363 matched disease-free controls.

Results: There was no evidence that imprinted gene methylation levels varied with age (between 25 and 87 years old), weight or height. Higher PEG3 methylation was associated with an elevated risk of IDC (odds ratio (OR) 1.065; $95 \%$ confidence interval (Cl) 1.002, 1.132; $p=0.042$ ) and DCIS (OR 1.139; $95 \%$ Cl 1.027, 1.263; $p=0.013)$. The effect was stronger when in situ and invasive breast cancer were combined (OR 1.079; $95 \% \mathrm{Cl} 1.020,1.142 ; p=0.008)$. DCIS breast cancer risk increased with higher KvDMR-ICR2 methylation (OR 1.395; $95 \%$ Cl 1.190, 1.635; $p<0.001$ ) and lower PLAGL1/ZAC1 methylation (OR 0.905; $95 \%$ Cl 0.833, 0.982; $p=0.017$ ). In a combined model, only KvDMR-ICR2 methylation remained significantly associated.
\end{abstract}

Conclusions: These findings may help to improve our understanding of the aetiology of breast cancer and the importance of early life factors in particular. Imprinting methylation status also has the potential to contribute to the development of improved screening and treatment strategies for women with, or at risk of, breast cancer.

Keywords: Imprinting, Methylation, Breast cancer, Invasive ductal carcinoma, Ductal carcinoma in situ

\section{Background}

Breast cancer is the most common type of cancer in women in the UK, with a lifetime risk of one in eight [1]. Improvements in detection and treatment have increased survival rates, and currently, $85 \%$ of women survive more than 5 years after diagnosis [1]. However, it is still the most common cause of cancer death in the world for women, with incidence markedly increasing [2]. The heritability of breast cancer is estimated at about $30 \%$ but known genetic risk variants (e.g. BRCA1, BRCA2, TP53 and PTEN) only account for around 5$10 \%$ of all cases [3, 4]. Non-genetic factors such as obesity, alcohol consumption, diet, birth weight and

\footnotetext{
* Correspondence: p.haggarty@abdn.ac.uk

'Division of Lifelong Health, Rowett Institute of Nutrition and Health, University of Aberdeen, Greenburn Road, Bucksburn, Aberdeen, UK Full list of author information is available at the end of the article
}

exposure to oestrogen have all been linked to breast cancer risk, but the mechanisms underpinning these associations have yet to be identified $[5,6]$.

A common observation in human breast cancers, and many other tumour types, is epigenetic change, including altered methylation of DNA. There is an increasing interest in the role of epigenetics as a potential mechanism linking environmental exposures to cancer risk and as a non-genetic explanation for cancer heritability [7]. Aberrant DNA methylation has been reported in breast tumours for a number of genes involved in apoptosis, cell cycle control and DNA repair [8]. One class of epigenetic mark with particular relevance to cancer is imprinting; the epigenetic marking of genes in a parent-of-origin specific manner within the germ cells [9]. There is intra-individual variation of imprinting DNA methylation, and the early factors that influence this variability and the potential 
effects on later life cancer risk are of particular interest [10-12]. Once set, many germ line imprints are maintained in a wide range of adult somatic tissues across the life course [13, 14]. A number of imprinted genes are known tumour suppressors or oncogenes, and loss of imprinting is a hallmark of many cancers $[8,15,16]$. Interestingly, relaxation or loss of imprinting in apparently normal tissue of individuals with cancer or those at increased risk of the disease has been observed in normal colonic epithelium and normal breast tissue $[17,18]$. These observations are relevant to the concept of field cancerisation-changes in normal tissue that predispose to the development of cancer-and the possible role of early factors in programming breast cancer susceptibility $[10,19]$. The soma-wide nature of imprinting also makes this signal useful to consider as a potential biomarker of breast cancer.

DNA methylation changes in blood have been reported in breast cancer [20-22] and in relation to the pathological characteristics of breast cancer including histological type, tumour size and receptor status [23, 24]. Some apparent changes in blood DNA methylation in disease states could simply reflect changes in blood cell types [25, 26], and one advantage of studying imprinted genes is that they are unaffected by cellular heterogeneity [27].

The present study was undertaken to determine whether breast cancer risk was associated with altered DNA methylation of selected imprinted differentially methylated regions (DMRs) in non-tumour tissue (blood). Six germ line DMRs, identified to regulate the associated imprinted gene regions, were chosen for pyrosequencing analysis: PLAGL1/ZAC1 [OMIM 603044], H19 [OMIM 103280], KvDMR [OMIM 604115], RB1 [OMIM 614041], SNRPN [OMIM 18227] and PEG3 [OMIM 601483]. A somatic region (DMR2) linked with IGF2 [OMIM 147470] was also selected for analysis. This DMR interacts with $H 19$ on the methylated paternal allele [28], has been linked with IGF2 expression [29] and has been associated with nutritional in utero exposures [12, 30] and birth outcomes [31]. Methylation status was determined in blood samples collected at diagnosis and prior to treatment from women with the most common type of invasive breast cancer (invasive ductal carcinoma-IDC), the most common type of in situ cancer (ductal carcinoma in situ-DCIS) and disease-free controls. Subjects were matched for age, height, weight, body mass index (BMI) and menopausal status. A growing number of studies investigating tumour and non-tumour DNA methylation levels use array technologies such as the Illumina Infinium ${ }^{\circ}$ HumanMethylation450 BeadChip [32] but this technology provides poor coverage of the imprinted regions of interest here. We investigated DNA methylation of these seven imprinted DMRs by pyrosequencing analysis.

\section{Results}

The mean methylation levels for all imprinted DMRs are summarised in Table 1 . These are in line with the predicted $50 \%$ DNA methylation levels for imprinted genes, $100 \%$ methylation on one allele and $0 \%$ on the other. The mean RB1 methylation was relatively high $(68.61 \%$; $\mathrm{SD}=4.65)$, but within the range of 33-70 \% methylation, commonly reported for imprinted regions [33]. The somatic imprinted region IGF2-DMR2 had the highest inter-individual variability $(48.50 \% ; \mathrm{SD}=5.72)$. There were no significant differences in age, height, weight and BMI between the matched cases and controls (Table 2).

The methylation level for the selected imprinted DMRs is shown for all of the disease-free women (Fig. 1). There was no evidence for a change in methylation of imprinted DMRs with age in this cross-sectional analysis. Neither were the imprinted DMR methylation levels significantly linked with weight or BMI of the disease-free women. Height was associated with $R B 1$ methylation, although this association was relatively weak (regression coefficient $=0.007 ; 95 \%$ CI 0.000 , 0.014; $p=0.049 ; r^{2}=0.0116$ ).

Increased PEG3 methylation was associated with an elevated risk of both IDC (odds ratio $=1.065 ; 95 \% \mathrm{CI}$ $1.002,1.132 ; p=0.042$ ) and DCIS (odds ratio $=1.139$; $95 \%$ CI 1.027, 1.263; $p=0.013)$ compared to the

Table 1 Average methylation levels for imprinted gene DMRs

\begin{tabular}{|c|c|c|c|c|c|c|c|}
\hline \multirow[t]{3}{*}{ Cohort } & \multicolumn{7}{|c|}{ Imprinted gene DMRs } \\
\hline & \multirow{2}{*}{$\begin{array}{l}\text { Chr. } 6 \\
\text { PLAGL1 }\end{array}$} & \multicolumn{3}{|l|}{ Chr. 11} & \multirow{2}{*}{$\begin{array}{l}\text { Chr. } 13 \\
\text { RBI }\end{array}$} & \multirow{2}{*}{$\begin{array}{l}\text { Chr. } 15 \\
\text { SNRPN }\end{array}$} & \multirow{2}{*}{$\begin{array}{l}\text { Chr. } 19 \\
\text { PEG3 }\end{array}$} \\
\hline & & H19 & IGF2 & KvDMR & & & \\
\hline Combined & $55.78(4.92)$ & $59.76(4.19)$ & $48.50(5.72)$ & $44.28(2.36)$ & $68.61(4.65)$ & $43.97(3.00)$ & $52.30(2.99)$ \\
\hline Disease-free & $55.91(4.92)$ & $59.59(4.35)$ & $48.15(5.77)$ & $44.17(2.34)$ & $68.61(4.30)$ & $44.03(3.03)$ & $52.05(2.93)$ \\
\hline DCIS & $53.90(3.60)$ & $59.70(3.79)$ & $49.52(5.66)$ & $45.81(1.99)$ & $68.22(3.95)$ & $43.62(3.20)$ & $53.29(3.61)$ \\
\hline IDC & $55.92(5.08)$ & $60.10(3.97)$ & $48.94(5.61)$ & $44.13(2.36)$ & $68.68(5.38)$ & $43.93(2.91)$ & $52.57(2.89)$ \\
\hline
\end{tabular}

Average percent methylation (\%) reported (and standard deviation). Combined cohort included all disease-free women and breast cancer cases $n=593$. Disease-free women $n=363$. DCIS (ductal carcinoma in situ) $n=41$. IDC (invasive ductal carcinoma) $n=189$

PLAGL1 pleomorphic adenoma gene like 1, H19 H19, imprinted maternally expressed non-coding transcript-imprint control region 1, IGF2 insulin-like growth factor 2. KVDMR $11 \mathrm{p} 15$ region imprint control region 2, RB1 retinoblastoma 1 . SNRPN small nuclear ribonucleoprotein polypeptide N, PEG3 paternally expressed gene 3. DMR: differentially methylated region. Chr: chromosome 
Table 2 Subject characteristics by disease status

\begin{tabular}{lllc}
\hline Characteristic & Controls $(n=363)$ & Cases $(n=230)$ & $p$ value \\
\hline Age $($ years $)$ & $56(11)$ & $56(11)$ & 0.499 \\
Height $(\mathrm{m})$ & $1.615(0.07)$ & $1.615(0.06)$ & 0.974 \\
Weight $(\mathrm{kg})$ & $70.91(14.25)$ & $70.75(13.50)$ & 0.889 \\
BMl $\left(\mathrm{kg} / \mathrm{m}^{2}\right)$ & $27.20(5.21)$ & $27.14(4.97)$ & 0.902 \\
\hline
\end{tabular}

Mean values with standard deviations (sd) shown in brackets. $p$ values calculated using a two-sample Student's $t$ test
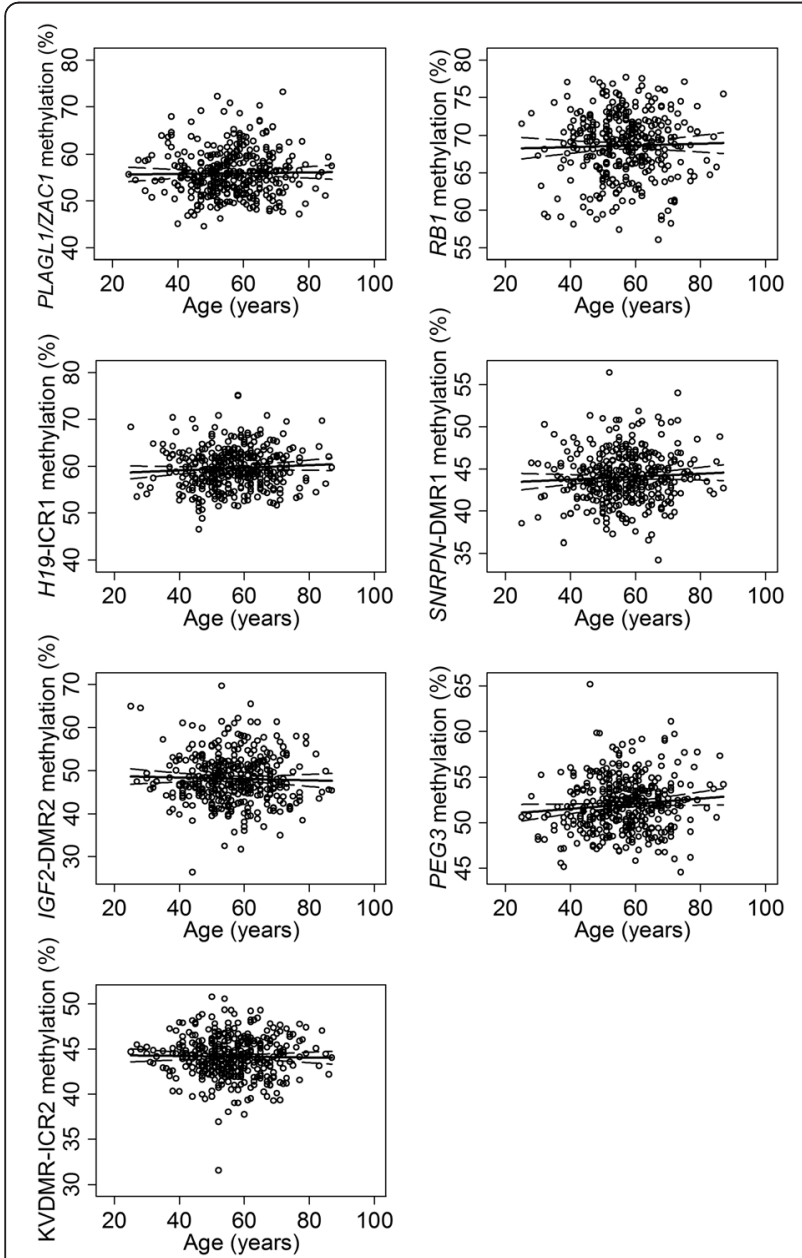

Fig. 1 Imprinted gene methylation and age in disease-free women. Average methylation (\%) within imprinted genes PLAGL1/ZAC1, RB1, H19-ICR1, SNRPN-DMR1, IGF2-DMR2, PEG3 and KvDMR-ICR2 according to age for all disease-free samples. Linear regression analysis fit shown with a solid line and with $95 \% \mathrm{Cl}$ shown with a dashed line. Average methylation levels are shown by an open circle. None of the associations were statistically significant. PLAGL1/ZAC1 pleomorphic adenoma gene like 1, RB1 retinoblastoma $1, H 19 \mathrm{H} 19$, imprinted maternally expressed non-coding transcript-imprint control region 1 , SNRPN small nuclear ribonucleoprotein polypeptide N, IGF2 insulinlike growth factor 2, PEG3 paternally expressed gene 3, KVDMR 11 p15 region imprint control region 2, Cl confidence intervals controls (Table 3). This effect was more significant when DCIS and IDC were combined (odds ratio $=1.079 ; 95 \%$ CI $1.020,1.142 ; p=0.008)$. There was no significant difference in PEG3 methylation between the IDC and DCIS cases. The distribution of PEG3 methylation according to disease status is shown in Fig. 2. IGF2 methylation was also higher in combined IDC and DCIS cases compared to controls but this effect did not achieve statistical significance $(p=0.068)$.

Two other DMRs exhibited differences in methylation in the DCIS cases compared to the controls (Table 3). Increased KvDMR-ICR2 methylation was associated with an elevated risk of DCIS (odds ratio $=1.395$; $95 \% \mathrm{CI}$ $1.190,1.635 ; p<0.001)$. Increased KvDMR-ICR2 methylation was also observed for the DCIS cases compared to the IDC cases (Odds Ratio $=1.413 ; 95 \%$ CI 1.168, 1.681; $p<0.001)$, confirming this association within DCIS cases only. Risk of DCIS decreased with increasing PLAGL1/ ZAC1 methylation (odds ratio $=0.905 ; 95 \%$ CI 0.833, $0.982 ; p=0.017$ ) (Table 3 ). Again, a significant difference was observed between the DCIS and IDC cases (odds ratio $=0.904 ; 95 \%$ CI 0.830, 0.983; $p=0.018$ ), confirming the effects for PLAGL1/ZAC1 methylation to be specific for the DCIS cases. Further statistical analysis showed that PLAGL1/ZAC1 methylation was inversely correlated with KVDMR-ICR2 (correlation coefficient $=-0.290 ; p<0.001)$. When both PLAGL1/ $Z A C 1$ and KVDMR-ICR2 were included in the same logistic regression model, only KvDMR-ICR2 remained significant (data not shown).

An overview of the pathological characteristics of IDC are summarised in Table 4. Further analysis based on subsets of the data produced some significant associations but the numbers in these comparisons were small and the results are included here primarily as hypothesis generating for future studies. Decreasing SNRPN methylation was associated with higher IDC histological grade (grade 3) compared to low histological grade 1 IDC (odds ratio $=0.808 ; 95 \%$ CI $0.674,0.969 ; p=0.022$ ) and combined histological grades 1 and 2 (odds ratio $=0.833$; $95 \%$ CI 0.711, 0.976; $p=0.024)$. Decreasing SNRPN methylation was also linked to increasing tumour size (regression coefficient $=-0.047 ; 95 \%$ CI $-0.076,-0.019$; $p=0.001)$. Both of these characteristics are associated with a poorer prognostic outcome. Higher SNRPN methylation was linked to a 'good' Nottingham Prognostic Index (NPI) prognostic category compared to a 'moderate,' 'poor' or combined 'moderate' and 'poor' category (data not shown). The NPI category is partly determined by IDC grade and tumour size. Increased KvDMR-ICR2 methylation appeared to be associated with oestrogen receptor (ER) positive status, after adjustment for breast cancer type (odds ratio $=1.197 ; 95 \%$ CI 1.032, 1.389; $p=0.018$ ). No other pathological features (including progesterone 
Table 3 Logistic regression analysis of the effect of methylation level of imprinted gene DMRs on disease risk

Imprinted gene DMRs

\begin{tabular}{|c|c|c|c|c|c|c|}
\hline \multirow{2}{*}{$\begin{array}{l}\text { Chr. } 6 \\
\text { PLAGL1/ZAC1 }\end{array}$} & \multicolumn{3}{|l|}{ Chr. 11} & \multirow{2}{*}{$\begin{array}{l}\text { Chr. } 13 \\
\text { RB1 }\end{array}$} & \multirow{2}{*}{$\begin{array}{l}\text { Chr. } 15 \\
\text { SNRPN-DMR1 }\end{array}$} & \multirow{2}{*}{$\begin{array}{l}\text { Chr. } 19 \\
\text { PEG3 }\end{array}$} \\
\hline & H19-ICR1 & IGF2-DMR2 & KvDMR-ICR2 & & & \\
\hline \multicolumn{7}{|l|}{ All Cases vs. Controls } \\
\hline $\begin{array}{l}0.987[0.953,1.022] \\
p=0.462\end{array}$ & $\begin{array}{l}1.026[0.986,1.068] \\
p=0.210\end{array}$ & $1.028[0.998,1.059] p=0.068$ & $1.049[0.976,1.128] p=0.193$ & $1.001[0.965,1.038] p=0.947$ & $0.985[0.930,1.043] p=0.601$ & $1.079^{* *}[1.020,1.142] p=0.008$ \\
\hline \multicolumn{7}{|l|}{ IDC vs. Controls } \\
\hline $\begin{array}{l}1.002[0.966,1.039] \\
p=0.932\end{array}$ & $\begin{array}{l}1.030[0.987,1.074] \\
p=0.171\end{array}$ & $1.025[0.994,1.057] p=0.123$ & $0.993[0.920,1.072] p=0.860$ & $1.006[0.967,1.046] p=0.772$ & $0.991[0.932,1.054] p=0.932$ & $1.065^{*}[1.002,1.132] p=0.042$ \\
\hline \multicolumn{7}{|l|}{ DCIS vs. Controls } \\
\hline $\begin{array}{l}0.905^{*}[0.833,0.982] \\
p=0.017\end{array}$ & $\begin{array}{l}1.007[0.932,1.088] \\
p=0.862\end{array}$ & $1.039[0.984,1.099] p=0.170$ & $1.395^{* * *}[1.190,1.635] p<0.001$ & $0.976[0.904,1.053] p=0.530$ & $0.954[0.851,1.068] p=0.412$ & $1.139 *[1.027,1.263] p=0.013$ \\
\hline \multicolumn{7}{|l|}{ DCIS vs. IDC } \\
\hline $\begin{array}{l}0.904^{*}[0.830,0.983] \\
p=0.018\end{array}$ & $\begin{array}{l}0.967[0.882,1.060] \\
p=0.474\end{array}$ & $1.021[0.961,1.084] p=0.509$ & $1.413^{* * *}[1.168,1.681] p<0.001$ & $0.984[0.924,1.048] p=0.613$ & $0.967[0.856,1.092] p=0.586$ & $1.084[0.971,1.270] p=0.150$ \\
\hline
\end{tabular}

Logistic regression analysis, reporting odds ratios [95\% confidence intervals]. ${ }^{*} p<0.05,{ }^{* *} p<0.01,{ }^{* * *} p<0.001$. Analysis adjusted for menopausal status, age and weight. All cases cohort describes combined IDC (invasive ductal carcinoma) and DCIS (ductal carcinoma in-situ) cases. DMR: differentially methylated region. Chr: chromosome. PLAGL1/ZAC1: Pleomorphic adenoma gene like 1. H19: H19, imprinted maternally expressed non-coding transcript-Imprint control region 1. IGF2: Insulin-like growth factor 2. KvDMR: 11 p15 region imprint control region 2. RB1: Retinoblastoma 1. SNRPN: Small nuclear Ribonucleoprotein polypeptide N. PEG3: Paternaly expressed 


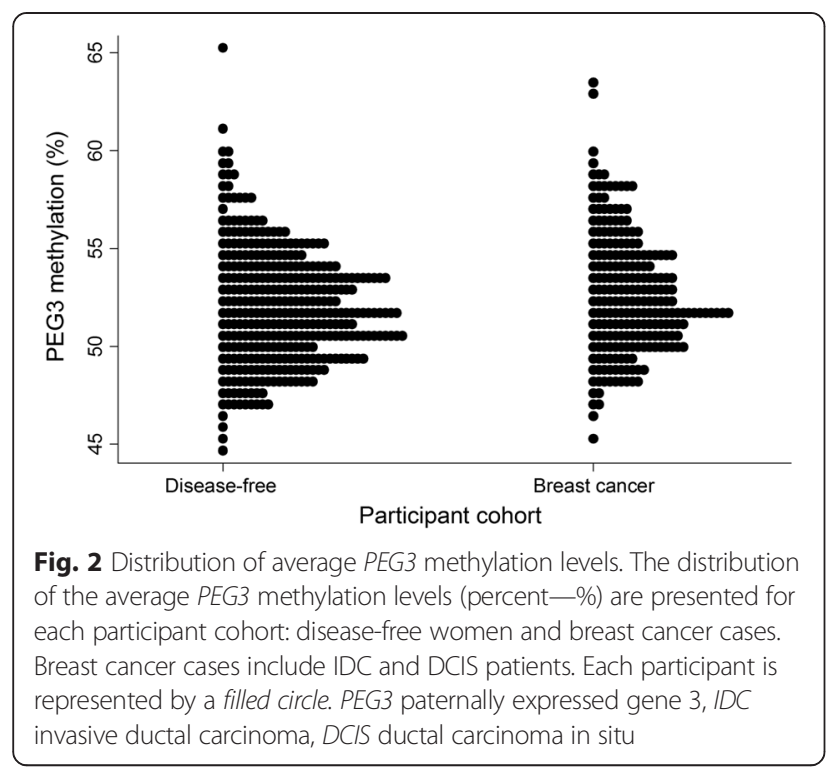

receptor-PR or human epidermal growth factor 2-HER2 status) were significantly associated with methylation levels.

\section{Discussion}

Cross-sectional analysis of all of the imprinted regions (PLAGL1/ZAC1, H19-ICR1, IGF2-DMR2, KvDMR-ICR2, RB1, SNRPN-DMR1 and PEG3) in the disease-free group indicated no change with age (between the age of 25 and 87 years). This reinforces the soma-wide nature of imprinting as blood cell type composition alters with age [34] which subsequently can influence methylation levels. These results support the view that imprinted regions are an attractive potential biomarker for examining breast cancer disease risk and progression, due to the stability of these throughout life [35].

Increased PEG3 methylation was associated with an elevated risk of both IDC and DCIS compared to the controls, and the level of significance was greater when the two types of cancer were combined. The findings for PEG3 in non-tumour tissue within this study mirror those previously reported in tumour samples: increased methylation of PEG3 has been observed in tumour tissue of invasive breast and ovarian cancers [36], as well as invasive cervical cancer [37]. Studies have confirmed correlation of PEG3 methylation between multiple tissue types, including blood and normal breast tissue [38] and that cancerous breast tumours have increased methylation compared to benign breast tumours [39]. PEG3 encodes a Kruppel-type zinc-finger protein which regulates the tumour necrosis factor (TNF) response, of which dysregulation is commonly implicated in cancer [OMIM 601483]. Aberrant PEG3 methylation in blood samples of women with breast cancer has recently been reported
Table 4 Pathological characteristics of IDC cases, according to menopausal status

\begin{tabular}{llll}
\hline Characteristic & $\begin{array}{l}\text { All cases } \\
(n=189)\end{array}$ & $\begin{array}{l}\text { Pre-menopausal } \\
(n=66)\end{array}$ & $\begin{array}{l}\text { Post-menopausal } \\
(n=123)\end{array}$ \\
\hline Grade & & & \\
Grade 1 & $23(12.2 \%)$ & $6(9 \%)$ & $17(13.8 \%)$ \\
Grade 2 & $84(44.4 \%)$ & $30(45.5 \%)$ & $54(43.9 \%)$ \\
Grade 3 & $81(42.9 \%)$ & $30(45.5 \%)$ & $51(41.5 \%)$ \\
Missing info. & $1(0.5 \%)$ & - & $1(0.8 \%)$
\end{tabular}

Lymph node involvement

$\begin{array}{llll}\text { None } & 119(63 \%) & 38(57.6 \%) & 81(65.9 \%) \\ \begin{array}{c}\text { Axillary lymph } \\ \text { nodes }\end{array} & 69(36.5 \%) & 28(42.4 \%) & 41(33.3 \%) \\ \text { Missing info. } & 1(0.5 \%) & - & 1(0.8 \%)\end{array}$

Hormone receptor status

$\begin{array}{llll}\text { ER positive } & 146 & 54(81.8 \%) & 92(74.8 \%) \\ \text { ER negative } & 43(22.8 \%) & 12(18.2 \%) & 31(25.2 \%) \\ \text { PR positive } & 145 & 53(80.3 \%) & 92(74.8 \%) \\ & (76.7 \%) & & \\ \text { PR negative } & 44(23.3 \%) & 13(19.7 \%) & 31(25.2 \%) \\ \text { HER2 receptor status } & & & \\ \text { Positive } & 22(11.6 \%) & 11(16.7 \%) & 11(9 \%) \\ \text { Negative } & 164 \%) & 54(81.8 \%) & 110(89.4 \%) \\ & (86.8 \%) & & 2(1.6 \%) \\ \text { Missing info. } & 3(1.6 \%) & 1(1.5 \%) & \end{array}$

Nottingham prognostic index (NPI)

$\begin{array}{llll}\text { Good (NPI } \leq 3.4) & 71(37.6 \%) & 20(30.3 \%) & 51(41.5 \%) \\ \begin{array}{l}\text { Moderate } \\ \text { (NPI 3.41-5.4) }\end{array} & 74(39.1 \%) & 32(48.5 \%) & 42(34.2 \%) \\ \text { Poor (NPI > 5.41) } & 35(18.5 \%) & 9(13.6 \%) & 26(21.1 \%) \\ \text { Missing info. } & 9(4.8 \%) & 5(7.6 \%) & 4(3.2 \%)\end{array}$

Participant numbers for pathological characteristics of IDC (invasive ductal carcinoma) patients overall and according to menopausal status (and percent-\% of each characteristic)

$E R$ oestrogen receptor, $P R$ progesterone receptor, HER2 human epidermal growth receptor 2

[39] but this analysis only highlighted the frequency of extreme outliers for PEG3 methylation, rather than the more subtle changes in average methylation reported here. The same study also reported differential outlier frequency of IGF2-DMR2 in blood samples [39]. Average IGF2-DMR2 methylation was also increased in combined cases compared to controls in the study described here, but this effect only approached statistical significance. There are also reports of altered IGF2 methylation in breast tumours [40].

Cancer marker studies suggest a common origin and development pathway of the two main types of breast cancer, IDC and DCIS. Reports have identified that lowgrade DCIS can transition to low-grade IDC and highgrade DCIS to high-grade IDC [41, 42]. The fact that 
PEG3 methylation in blood is associated with both DCIS and IDC separately in this study suggests that this change could contribute to the common origin and development pathway. This information could potentially contribute to the development of early biomarkers of breast cancer susceptibility.

Not all women with DCIS will go on to develop invasive breast cancer. The risk of progression is estimated at $25-50 \%$ for women with low-grade DCIS and $75 \%$ for women with high-grade DCIS [43]. A recent review suggested that screening asymptomatic women by mammography resulted in a $20 \%$ reduction in mortality, but the authors also concluded that around $19 \%$ of diagnosed cancers would not have caused a problem if left untreated [44] and others have suggested that there may be no survival advantage by breast screening, with the treatment itself being associated with a significant net morbidity and mortality [2].

An important clinical challenge is to identify which individuals can safely be left without treatment [45]. This study identified DNA methylation changes in blood samples associated with DCIS only. Increased KvDMR-ICR2 methylation and decreased PLAGL1/ZAC1 methylation were linked with increasing risk of DCIS compared to both controls and IDC cases. When both genes were included in the same statistical model, only the significance for KvDMR-ICR2 methylation remained. Such findings have the potential to identify individuals with DCIS who are less likely to progress to invasive breast cancer. KvDMR-ICR2 regulates the CDKN1C/KCNQ1 domain, including the non-coding antisense KCNQ1OT1 RNA which silences genes on the paternal allele within this region. Downregulation of CDKN1C (which encodes $\mathrm{p} 57(\mathrm{KIP} 2)$, a negative regulator of cell proliferation-OMIM 600856) has been associated with increased metastasis potential and poorer survival for breast cancer [46, 47]. Decreased KCNQ1OT1 methylation has been identified in tumour development and the imprinting disorder Beckwith-Wiedemann syndrome which is associated with tumour predisposition [48]. Altered KvDMR-ICR2 methylation, as observed within this study, may influence breast cancer risk through an effect on CDKN1C or KCNQ1OT1. Further work on the links between KvDMR-ICR2 methylation and $C D K N 1 C / K C N Q 1 O T 1$ gene expression in cancer would be instructive.

Links between imprint signals in normal tissue (such as blood) and breast cancer risk are particularly relevant to the concept of 'field cancerisation' [19]. This is based on the idea that widespread changes in normal tissues precede the development of the disease and increase the risk of transition to cancer. There is a renewed interest in this paradigm in relation to epigenetic change, and it is particularly relevant to imprinting as many germ line DMRs stably maintain their allele specific methylation signal in a wide range of adult somatic tissues over the decades $[38,49,50]$. The soma-wide nature of certain imprinting signals, such as those studied here, makes them particularly convenient as biomarkers of breast cancer risk, type and prognosis since the signal should be detectable in easily accessible tissues such as blood. Cell-free circulating DNA (cfDNA) has also been used in epigenetic studies but these applications tend to be used for metastatic breast cancer [51]. The correlation between some imprinting methylation in tumour and normal tissue means that cfDNA originating from tumours could also potentially be used for this type of work but there is an extensive epigenetic change in tumours, and cfDNA may not always be relevant to investigations of cancer-predisposing epigenetic change in normal tissues.

The differences in imprinting methylation described here are also relevant to observations linking breast cancer susceptibility to early development [10]. A number of studies and meta-analyses have demonstrated a clear relationship between birth weight and breast cancer risk, though there is a debate as to the relative strength of the association for pre- and post-menopausal breast cancer [52-56]. The mechanism linking birth weight to breast cancer risk has not yet been established but imprinting could provide a plausible link, as one of the key functions of imprinted genes is to regulate foetal growth [57]. Imprinting methylation has also been linked to birth weight and health of the new born [31].

Interestingly, there is some evidence that methylation of imprinted genes may be modifiable by the early environment such as maternal nutrition before birth. One example of this is folic acid. This nutrient is metabolised through the pathway that provides methyl groups for DNA methylation, and its use in human pregnancy has been reported to influence PEG3 and IGF2 methylation in the offspring [12]. The methylation differences observed here are relatively small in magnitude, and this is generally the case in population epigenetic studies linking average methylation levels to phenotypes $[10,31,58]$. Human epigenetic studies almost universally measure the average methylation level in a sample of cells, usually blood or buccal cells, but the distribution and variance of methylation states may be more important [58]. Many human diseases have the potential to develop from a subset of cells, or even one cell as in cancer, and small changes in the average methylation level could reflect more important changes in the proportion of cells in high-risk epigenotype states [56]. From this study, we are not able to suggest a clear cut-off threshold to identify those individuals who will definitely develop breast cancer, but the measurement denotes statistical probability. We suggest that the significant regions are worth looking at in more depth to develop a more detailed picture of the methylation signal 
using methods such as next-generation bisulphite sequencing and that they may be used in conjunction with other risk factors to improve the overall predictive value of these imprinted gene regions.

\section{Conclusions}

Altered DNA methylation has been shown to occur in tumours, with changes now being identified in nontumour tissue such as blood samples. Imprinted gene methylation is particularly interesting to consider as these provide potential mechanistic routes to early life influences and non-genetic heritability of breast cancer. Using a case-control cohort of almost 600 women, we have observed increased PEG3 methylation within blood samples of both invasive and in situ breast cancer cases. Increased KvDMR-ICR2 methylation was observed in women with in situ breast cancer compared to both controls and invasive breast cancers. DNA methylation stability was confirmed for the seven selected imprinted gene regions within this cohort. The findings set out in this study suggest potential biomarkers for breast cancer risk.

\section{Methods}

\section{Study population}

The study was approved by the North of Scotland Research Ethics Committee (reference number 08/S0801/ 17), and all participants gave informed consent. Two thousand and one hundred seventy-two women were recruited between 2008 and 2013 from the Aberdeen Breast Clinic with 1168 breast cancer cases and 1004 disease-free controls. The breast cancer patients were newly diagnosed, with histological confirmation and without detectable metastatic disease. Blood samples were taken prior to surgical intervention. The diseasefree women had attended the Aberdeen Breast Clinic with benign breast changes, none of which significantly increased their risk of breast cancer. Disease-free status was confirmed by clinical examination, mammography, breast ultrasonography and fine needle aspiration cytology where appropriate.

Only European Caucasians were included in the study and women with known family history of $B R C A 1 / 2 \mathrm{mu}-$ tations and those which have been identified at an increased risk of breast cancer were excluded from the analysis. The women were identified as having an increased risk of breast cancer if there was known family history, previous occurrence of atypical ductal hyperplasia, lobular carcinoma in situ or Hodgkin's disease.

Participant characteristics and pathological information including histological type, tumour size, tumour grade, lymph node status, hormone receptor status and HER2 expression status were recorded. Nottingham Prognostic Index was determined using tumour grade, tumour size and involvement of lymph nodes [59]. In the initial phase of the study, we included all types of cancer in the case group and matched an equivalent numbers of controls. After applying the study exclusion criteria and removing the more unusual cancer types (numbers too small for meaningful comparison), the number of controls $(n=363)$ exceeded the number of cases $(n=230)$ but the groups remained well matched for the key variables of age, height, weight, body mass index (BMI) and menopausal status.

\section{Participant characteristics}

Age, height, weight and BMI of the participants are summarised in Table 2. The DCIS cases were classified as intermediate $(27 \%)$, intermediate/high (17\%) or high (56\%) grade cancers. Information on ER status was available for $78 \%$ of the DCIS cases (of these, $84 \%$ were positive and $16 \%$ negative) and PR status for $76 \%$ of the DCIS cases (of these, $74 \%$ were positive and $26 \%$ negative). Pathological characteristics for the IDC cases are summarised in Table 4.

\section{DNA methylation analysis}

DNA was extracted from the blood using QIAamp DNA Mini Blood QIAcube kits (Qiagen, Crawley, UK), automated on a QIAcube (Qiagen, Crawley, UK). The DNA was quantified with $\mathrm{SYBR}^{\circ}$ Green on a Rotergene Q (Qiagen, Crawley, UK) using DNA standards (Life Technologies, Paisley, UK). The DNA samples were dispensed into 96-well plates by a QIAgility robotic system (Qiagen, Crawley, UK). Matched samples (breast cancer cases and disease-free controls) were positioned on the same plate to minimise any potential batch effects. The samples were treated with sodium bisulphite using Epitect 96 Bisulfite kits (Qiagen, Crawley, UK). Published PCR primers designed for bisulphite-converted DNA were used for PLAGL1/ZAC1, IGF2-DMR2, SNRPNDMR1 and PEG3 assays [60-63]. FASTA sequences were used to design specific assays using PyroMark Assay Design Software (version 2.0, Qiagen, Crawley, UK) for the H19-ICR1, KvDMR-ICR2 and RB1 assays [33]. The primers were as follows: forward 5'-TGGGGATTTTG ATGGGGTTA-3', reverse 5' -biotin-CCTACTCCAAA CATTATAAAAAAAACTAAC-3' and sequencing primer 5'-GATGGTTAGGGTGTGTT-3' for the H19-ICR1 assay; forward 5 '-biotin-GGGTGATTATTGGAGTTG TTGAGGTGAG-3', reverse $5^{\prime}$-TCCAATCCCAATTC AACCCACTC-3' and sequencing primer 5'-CTAA ACCACCATAAAAACTAT-3' for the KvDMR-ICR2 assay; forward 5' -biotin-TGGGGTTAGGAGGTGAAA GTGG-3', reverse 5' -CATATAAAACAACAACAAAT CCCTTTCTAC-3' and sequencing primer $5^{\prime}$-CCCT AAACCTACCTTCCC-3' for the RB1 assay. Pyrosequencing was performed using the PyroMark MD Q96 
Instrument (Pyrosequencing, Inc., Uppsala, Sweden) running PyroMark CpG software (version 1.0.9, Biotage GB Ltd., Hengoed, UK). The plates were prepared for pyrosequencing according to the manufacturers' protocol. Only data which passed appropriate quality control thresholds were included for analysis.

\section{Statistical analysis}

Data were analysed using STATA/SE version 13 (Stata Corp, College Station, Texas, USA). Subject characteristics of the entire cohort and pathological characteristics of breast cancer cases were compared using Student's $t$ test. Pairwise correlation was run to determine the relationship between $\mathrm{CpG}$ sites within each gene/region. CpG sites within each gene (PEG3 and RB1; seven CpG sites H19-ICR1, KvDMR-ICR2 and PLAGL1; six CpG sites SNRPN and IGF2; four CpG sites each) were highly correlated with each other (all at $p<0.01$ ), and the average methylation for each of these genes/regions was used in subsequent analysis. Linear regression was used to determine the relationship between methylation and age, weight, height and body mass index in the diseasefree control group.

Logistic regression was used to compare methylation levels in the cases (IDC only, DCIS only and IDC and DCIS combined) relative to the disease-free controls. IDC was also compared with DCIS. Logistic regression was used for other categorical outcomes (lymph node involvement and receptor status). Multinomial logistic regression was used to evaluate the link between methylation and NPI categories. All analyses were adjusted for appropriate covariates including menopausal status, age and weight (as stated in the tables and text). The results of regression analysis are presented with $95 \%$ confidence intervals and $p$ values. Significance was set at $" p<0.05,{ }^{* * *} p<0.01$ and ${ }^{* * *} p<0.001$. Due to the severe effect of multiple comparison adjustments on false negative error rates and the exploratory nature of this research, such adjustments have been avoided to prevent this.

\section{Competing interests}

The authors declare that they have no competing interests.

\section{Authors' contributions}

$\mathrm{KH}$ carried out the DNA extractions, assay design, methylation analysis and statistical analysis and drafted the manuscript. GH carried out the bisulphite conversions. PS and LS recruited the participants, collected the samples and collated all participant data. GWH contributed statistical advice for the study design and during statistical analysis. ES participated in the design and recruitment of the study. SDH conceived the study, participated in the design, collected participant data and helped to draft the manuscript. PH conceived the study, participated in the design and coordination, provided statistical support and helped to draft the manuscript. All authors have read and approved the final manuscript.

\section{Acknowledgements}

This study was supported by the Breast Cancer Campaign (2008MayPR46) and Fraserburgh Moonlight Prowl Breast Cancer Charity. PH, GH and GWH acknowledge the support of the Scottish Government. We would like to thank Val Bain and Michela Donnarumma for help with the data and sample collection.

\section{Author details}

${ }^{1}$ Division of Lifelong Health, Rowett Institute of Nutrition and Health, University of Aberdeen, Greenburn Road, Bucksburn, Aberdeen, UK. ${ }^{2}$ Division of Applied Medicine, School of Medicine and Dentistry, University of Aberdeen, University Medical Buildings, Foresterhill, Aberdeen, UK.

${ }^{3}$ Biomathematics and Statistics Scotland, Aberdeen, UK. ${ }^{4}$ Aberdeen Royal Infirmary, Ward 308, Foresterhill, Aberdeen, UK.

Received: 20 June 2015 Accepted: 17 August 2015

Published online: 04 September 2015

\section{References}

1. Cancer Research UK [http://www.cancerresearchuk.org/health-professional/ cancer-statistics/statistics-by-cancertype/breast-cancer]

2. Cancer Incidence and Mortality Worldwide: IARC CancerBase No. 11. International Agency for Research on Cancer; 2013. [http://globocan.iarc.fr]

3. Claus EB, Schildkraut JM, Thompson WD, Risch NJ. The genetic attributable risk of breast and ovarian cancer. Cancer. 1996;77(11):2318-24.

4. Locatelli I, Lichtenstein P, Yashin Al. The heritability of breast cancer: a Bayesian correlated frailty model applied to Swedish twins data. Twin Res. 2004;7(2):182-91.

5. Cottet V, Touvier M, Fournier A, Touillaud MS, Lafay L, Clavel-Chapelon F, et al. Postmenopausal breast cancer risk and dietary patterns in the E3N-EPIC prospective cohort study. Am J Epidemiol. 2009;170(10):1257-67.

6. Wolff MS, Collman GW, Barrett JC, Huff J. Breast cancer and environmental risk factors: epidemiological and experimental findings. Annu Rev Pharmacol Toxicol. 1996;36(1):573-96.

7. Szyf M, Pakneshan P, Rabbani SA. DNA methylation and breast cancer. Biochem Pharmacol. 2004;68(6):1187-97.

8. Cheung HH. DNA methylation of cancer genome. Birth Defects Res A Clin Mol Teratol. 2009;87(4):335.

9. Reik W. Epigenetic reprogramming in mammalian development. Science. 2001;293(5532):1089.

10. Haggarty P, Heys SD: Nutrition and development: short- and long-term consequences for health, ed. In Nutrition and Development: Cancer. Edited by Sanders TAB. West Sussex, UK: Wiley-Blackwell; 2013:164-176

11. Heijmans BT, Kremer D, Tobi EW, Boomsma DI, Slagboom PE. Heritable rather than age-related environmental and stochastic factors dominate variation in DNA methylation of the human IGF2/H19 locus. Hum Mol Genet. 2007;16(5):547-54.

12. Haggarty P, Hoad G, Campbell DM, Horgan GW, Piyathilake C, McNeill G. Folate in pregnancy and imprinted gene and repeat element methylation in the offspring. Am J Clin Nutr. 2013:97(1):94-9.

13. Woodfine K, Huddleston JE, Murrell A. Quantitative analysis of DNA methylation at all human imprinted regions reveals preservation of epigenetic stability in adult somatic tissue. Epigenetics Chromatin. 2011;4(1):1.

14. Coolen MW, Statham AL, Qu W, Campbell MJ, Henders AK, Montgomery GW, et al. Impact of the genome on the epigenome is manifested in DNA methylation patterns of imprinted regions in monozygotic and dizygotic twins. PLoS One. 2011;6(10):e25590.

15. Uribe-Lewis S, Woodfine K, Stojic L, Murrell A. Molecular mechanisms of genomic imprinting and clinical implications for cancer. Expert Rev Mol Med. 2011;13:e2.

16. Yuasa Y. DNA methylation in cancer and ageing. Mech Ageing Dev. 2002;123(12):1649-54.

17. Feinberg AP, Ohlsson R, Henikoff S. The epigenetic progenitor origin of human cancer. Nat Rev Genet. 2006;7(1):21-33.

18. Hu M, Yao J, Cai L, Bachman KE, van den Brûle F, Velculescu V, et al. Distinct epigenetic changes in the stromal cells of breast cancers. Nat Genet. 2005;37(8):899-905.

19. Slaughter DP, Southwick HW, Smejkal W. Field cancerization in oral stratified squamous epithelium; clinical implications of multicentric origin. Cancer. 1953;6:963-8.

20. Xu Z, Bolick SC, DeRoo LA, Weinberg CR, Sandler DP, Taylor JA. Epigenome-wide association study of breast cancer using prospectively collected sister study samples. J Natl Cancer Inst. 2013;105(10):694-700.

21. Choi JY. Association between global DNA hypomethylation in leukocytes and risk of breast cancer. Carcinogenesis. 2009;30(11):1889. 
22. Wu H, Delgado-Cruzata L, Flom JD, Perrin M, Liao Y, Ferris JS, et al. Repetitive element DNA methylation levels in white blood cell DNA from sisters discordant for breast cancer from the New York site of the Breast Cancer Family Registry. Carcinogenesis. 2012;33(10):1946-52.

23. Fleischer T, Edvardsen H, Jovanovic J, Touleimat N, Børresen-Dale A, Tost J, et al. DNA methylation and gene expression patterns in breast cancer progression from in situ carcinoma to invasive carcinoma. Epigenetics Chromatin. 2013;6 Suppl 1:18.

24. Fackler MJ. Genome-wide methylation analysis identifies genes specific to breast cancer hormone receptor status and risk of recurrence. Cancer Res. 2011;71(19):6195.

25. Reinius LE, Acevedo N, Joerink M, Pershagen G, Dahlén S, Greco D, et al. Differential DNA methylation in purified human blood cells: implications for cell lineage and studies on disease susceptibility. PLoS One. 2012;7(7):e41361.

26. Adalsteinsson BT, Gudnason H, Aspelund T, Harris TB, Launer LJ, Eiriksdottir $\mathrm{G}$, et al. Heterogeneity in white blood cells has potential to confound DNA methylation measurements. PLoS One. 2012;7(10):e46705.

27. Talens RP, Boomsma DI, Tobi EW, Kremer D, Jukema JW, Willemsen G, et al. Variation, patterns, and temporal stability of DNA methylation: considerations for epigenetic epidemiology. FASEB J. 2010;24(9):3135-44.

28. Tollefsbol T: Epigenetics in human disease. Massachusetts, USA: Academic Press; 2012.

29. Dejeux E, Olaso R, Dousset B, Audebourg A, Gut IG, Terris B, et al. Hypermethylation of the IGF2 differentially methylated region 2 is a specific event in insulinomas leading to loss-of-imprinting and overexpression. Endocr Relat Cancer. 2009;16(3):939-52.

30. Soubry A, Schildkraut JM, Murtha A, Wang F, Huang Z, Bernal A, et al. Paternal obesity is associated with IGF2 hypomethylation in newborns: results from a Newborn Epigenetics Study (NEST) cohort. BMC Med. 2013;11:29-7015. 11-29.

31. Haggarty P, Hoad G, Horgan GW, Campbell DM. DNA methyltransferase candidate polymorphisms, imprinting methylation, and birth outcome. PLoS One. 2013;8(7):e68896.

32. Dedeurwaerder S, Defrance M, Calonne E, Denis H, Sotiriou C, Fuks F. Evaluation of the Infinium Methylation 450K technology. Epigenomics. 2011;3(6):771-84.

33. Das R, Lee YK, Strogantsev R, Jin S, Lim YC, Ng PY, et al. DNMT1 and AIM1 Imprinting in human placenta revealed through a genome-wide screen for allele-specific DNA methylation. BMC Genomics. 2013;14(1):685.

34. Jaffe $A E$, Irizarry RA. Accounting for cellular heterogeneity is critical in epigenome-wide association studies. Genome Biol. 2014;15(2):R31.

35. Murphy SK, Huang Z, Hoyo C. Differentially methylated regions of imprinted genes in prenatal, perinatal and postnatal human tissues. PLoS One. 2012;7(7):e40924.

36. Huang JM, Kim J. DNA methylation analysis of the mammalian PEG3 imprinted domain. Gene. 2009;442(1-2):18-25.

37. Nye MD, Hoyo C, Huang Z, Vidal AC, Wang F, Overcash F, et al. Associations between methylation of paternally expressed gene 3 (PEG3), cervical intraepithelial neoplasia and invasive cervical cancer. PLoS One. 2013;8(2):e56325.

38. Woodfine K, Huddleston JE, Murrell A: Quantitative analysis of DNA methylation at all human imprinted regions reveals preservation of epigenetic stability in adult somatic tissue. Epigenetics and Chromatin 2011;4(1):1-13.

39. Barrow TM, Barault L, Ellsworth RE, Harris HR, Binder AM, Valente AL, Shriver CD, Michels KB: Aberrant methylation of imprinted genes is associated with negative hormone receptor status in invasive breast cancer. International Journal of Cancer 2015

40. van Roozendaal C, Gillis A, Klijn J, van Ooijen B, Claassen C, Eggermont A, et al. Loss of imprinting of IGF2 and not H19 in breast cancer, adjacent normal tissue and derived fibroblast cultures. FEBS Lett. 1998;437(1-2):107-11.

41. Bombonati A, Sgroi DC. The molecular pathology of breast cancer progression. J Pathol. 2011;223(2):308-18.

42. Leonard GD, Swain SM. Ductal carcinoma in situ, complexities and challenges. J Natl Cancer Inst. 2004;96(12):906-20.

43. Evans AJ, Pinder SE, Ellis IO, Wilson ARM. Screen detected ductal carcinoma in situ (DCIS): overdiagnosis or an obligate precursor of invasive disease? J Med Screen. 2001;8(3):149-51.

44. Independent UK Panel on Breast Cancer Screening. The benefits and harms of breast cancer screening: an independent review. Lancet. 2012;380(9855):1778
45. Virnig BA, Tuttle TM, Shamliyan T, Kane RL. Ductal carcinoma in situ of the breast: a systematic review of incidence, treatment, and outcomes. J Natl Cancer Inst. 2010;102(3):170-8.

46. Yang X, Karuturi RM, Sun F, Aau M, Yu K, Shao R, et al. CDKN1C (p57KIP2) is a direct target of EZH2 and suppressed by multiple epigenetic mechanisms in breast cancer cells. PLoS One. 2009;4(4):e5011.

47. Xu X, Wang W, Zhang L, Li Y, Tang M, Jiang N, et al. Clinical implications of p57KIP2 expression in breast cancer. Asian Pac J Cancer Prev. 2012;13(10):5033-6.

48. Weksberg R, Nishikawa J, Caluseriu O, Fei Y, Shuman C, Wei C, et al. Tumor development in the Beckwith-Wiedemann syndrome is associated with a variety of constitutional molecular 11p15 alterations including imprinting defects of KCNQ1OT1. Hum Mol Genet. 2001;10(26):2989-3000.

49. Heaphy CM, Griffith JK, Bisoffi M. Mammary field cancerization: molecular evidence and clinical importance. Breast Cancer Res Treat. 2009;118(2):229-39.

50. Sandovici I, Leppert M, Hawk PR, Suarez A, Linares Y, Sapienza C. Familial aggregation of abnormal methylation of parental alleles at the IGF2/H19 and IGF2R differentially methylated regions. Hum Mol Genet. 2003;12(13):1569-78.

51. Fackler MJ, Lopez Bujanda Z, Umbricht C, Teo WW, Cho S, Zhang Z, et al. Novel methylated biomarkers and a robust assay to detect circulating tumor DNA in metastatic breast cancer. Cancer Res. 2014;74(8):2160-70.

52. Ahlgren M, Sørensen T, Wohlfahrt J, Haflidadóttir Á, Holst C, Melbye M. Birth weight and risk of breast cancer in a cohort of 106,504 women. Int J Cancer. 2003;107(6):997-1000.

53. Forman MR, Cantwell MM, Ronckers C, Zhang Y. Through the looking glass at early-life exposures and breast cancer risk. Cancer Invest. 2005;23(7):609-24.

54. De Stavola BL, Hardy R, Kuh D, dos Santos SI, Wadsworth M, Swerdlow AJ. Birth weight, childhood growth and risk of breast cancer in a British cohort. Br J Cancer. 2000:83(7):964.

55. Okasha M, McCarron P, Gunnell D, Smith GD. Exposures in childhood, adolescence and early adulthood and breast cancer risk: a systematic review of the literature. Breast Cancer Res Treat. 2003;78(2):223-76.

56. dos Santos SI, De Stavola B, McCormack V. Birth size and breast cancer risk: re-analysis of individual participant data from 32 studies. PLoS Med. 2008;5(9):e193.

57. Azzi S, Sas TC, Koudou Y, Le Bouc Y, Souberbielle J, Dargent-Molina P, et al Degree of methylation of ZAC1 (PLAGL1) is associated with prenatal and post-natal growth in healthy infants of the EDEN mother child cohort. Epigenetics. 2013;9(3).

58. Haggarty P. Nutrition and the epigenome. Prog Mol Biol Transl Sci. 2012;108:427-46

59. Elston C, Ellis I. Pathological prognostic factors in breast cancer. I. The value of histological grade in breast cancer: experience from a large study with long-term follow-up. Histopathology. 1991;19(5):403-10.

60. Bourque DK, Avila L, Peñaherrera M, von Dadelszen P, Robinson WP. Decreased placental methylation at the H19/IGF2 imprinting control region is associated with normotensive intrauterine growth restriction but not preeclampsia. Placenta. 2010;31(3):197-202.

61. Dupont J, Tost J, Jammes H, Gut IG. De novo quantitative bisulfite sequencing using the pyrosequencing technology. Anal Biochem. 2004;333(1):119-27.

62. White HE, Durston VJ, Harvey JF, Cross NCP. Quantitative analysis of SRNPN gene methylation by pyrosequencing as a diagnostic test for Prader-Willi syndrome and Angelman syndrome. Clin Chem. 2006;52(6):1005-13.

63. Feng W, Marquez RT, Lu Z, Liu J, Lu KH, Issa J-J, et al. Imprinted tumor suppressor genes ARHI and PEG3 are the most frequently down-regulated in human ovarian cancers by loss of heterozygosity and promoter methylation. Cancer. 2008;112(7):1489-502. 